\title{
FGFR2IIIb Positive
}

National Cancer Institute

\section{Source}

National Cancer Institute. FGFR2IIIb Positive. NCI Thesaurus. Code C142105.

An indication that FGFR2IIIb expression has been detected in a sample. 